\title{
Diagnostic Value of Bedside Ultrasound in Intracranial Hemorrhage of High-Risk Neonates
}

\author{
Li Kai-Wen, Gong Lan
}

Department of Ultrasound, Jingzhou Central Hospital,The Second Clinical Medical College, Yangtze University, Jingzhou, China

Email address:

likaiwen1234@163.com (Li Kai-Wen),gong318@sina.com (Gong Lan)

\section{To cite this article:}

Li Kai-Wen, Gong Lan. Diagnostic Value of Bedside Ultrasound in Intracranial Hemorrhage of High-risk Neonates. International Journal of Biomedical Engineering and Clinical Science. Vol. 3, No. 3, 2017, pp. 18-21. doi: 10.11648/j.ijbecs.20170303.11

Received: March 11, 2017; Accepted: April 5, 2017; Published: October 18, 2017

\begin{abstract}
To investigate diagnostic value of Bedside Ultrasound and computed tomography (CT) for detecting intracranial hemorrhage in newborn, 1750 neonates in our department of newborn intensive care unit were examined with Bedside Ultrasound and CT. After ultrasonography diagnosed 377 intracranial hemorrhage, it can be found that in premature infants the incident of intracranial hemorrhage was higher than term infants $(\mathrm{P}<0.001)$, and the incident of periventricle-intraventricle hemorrhage (PVH-IVH) I grade was significantly higher than grade II, III, IV $(\mathrm{P}=0.000)$. 41 cases grade I PVH-IVH were confirmed by Bedside Ultrasound, but negative in CT scan. It can be concluded that bedside Ultrasound have a very important clinical value to increase the incident of newborn intracranial hemorrhage, especially PVH-IVH grade I.
\end{abstract}

Keywords: Diagnostic Value, Bedside Ultrasound, Intracranial Hemorrhage, Neonates

\section{Introduction}

The common neurological disease in the newborn is intracranial hemorrhage, of which the most common is periventricular-intravent-ricular hemorrhage (PVH-IVH), that's the common reason why high-risk newborns are dead or nervous-system damage. So early diagnosis and timely treatment of neonatal intracranial hemorrhage has important clinical significance. In this study, 1750 cases of high-risk neonates who were hospitalized in our hospital from January 2011 to January 2017 were selected and examined by bedside brain ultrasonography from 3days to 7 days after birth. The results were compared with those of CT examinations. The clinical value of paravertebral ultrasonography in the diagnosis of intracranial hemorrhage in high-risk neonates is reported below.

\section{Materials and Methods}

\subsection{General Information}

1750 cases of high-risk neonates who were hospitalized in our hospital from January 2011 to January 2017 were selected, including 907 males and 843 females, aged $26.7 \sim 41.6$ weeks, average $(32.6 \pm 2.5)$ weeks, 1315 cases of premature infants (gestational age $<37$ weeks), 435 cases of full-term infants (gestational age $\geq 37$ weeks). High-risk infants are the babies affected by a variety of high-risk factors that occur or may occur dangerous situations. The conditions include high risk factors of pregnant women, the birth process, fetuses and newborns [1]. Therefore, the selection criteria should include at least one of the following conditions: birth weight $<2500 \mathrm{~g}$, premature infants, suspected intracranial hemorrhage, suspected hypoxic ischemic encephalopathy, persistent hypoxemia, persistent hypoglycemia, hemolytic disease, hyperbilirubinemia, multiple births, intrauterine distress, and pregnant women who have a high risk of pregnancy and other medical history.

\subsection{Methods}

All the high-risk infants were examined by bedside brain ultrasonography from 3 days to 7 days after birth, and the results were compared with CT reports.

\subsubsection{Instruments and Inspection Methods}

Using the Marei M7 portable color Doppler ultrasound diagnostic instrument, and select the probe frequency of 8.0 $\mathrm{MHz}$ which is appropriative ultrasound probe (C8-5) for newborn brains. Check the child when asked to be quiet or sleeping, those who do not cooperate should have pacifiers for comforting. The patients should be supine, the halo indwelling 
needle and other covers which may interfere should be removed before scanning. To take the parts of the coronal plane and sagittal plane according to the standard cut. On the basis of the above-mentioned section, we can scan from any angle, so that most of the brain can be scanned as much as possible. Recording the size of the bleeding lesions and tell whether the echo and the border is clear, describing the brain parenchymal echo, collecting important intracranial structure of the negative, positive sonogram and store them. Positive children or ultrasound found no bleeding but clinically suspected cases of hemorrhage should do CT examinations [2]. While checking, we should keep the probe clean, and use medical disinfection ultrasonic coupling agent to avoid cross-infection. Spiral CT scan should apply the United States GE Bright Speed 16-slice spiral CT, conventional adjustment layer thickness $1 \mathrm{~mm}$, pitch $1 \mathrm{~mm}$, using the skull scan to obtain images.

\subsubsection{Papile Grading Methods}

Intracranial hemorrhage is generally divided into the following types, namely, Periventricular - Intraventricular Hemorrhage (PVH-IVH), Subdural hemorrhage, Subarachnoid Hemorrhage, Cerebellar Hemorrhage. Among which PVH-IVH Is usually divided into the following four according to Papile Grading Methods [3-4]

(1) Grade I: Subependymal germinal matrix hemorrhage can occur in one side or both.

(2) Grade II: Intraventricular hemorrhage (due to subependymal hemorrhage caused by perforation ependymal), but there is no ventricular dilatation.

(3) Grade Ш: Intraventricular hemorrhage with ventricular dilatation. The measurement method is to measure the widest longitudinal diameter of the lateral ventricle in the parietal plane, $6 \sim 10 \mathrm{~mm}$ for mild ventricular dilatation, $11 \sim 15 \mathrm{~mm}$ for moderate ventricular dilatation, $>15 \mathrm{~mm}$ for severe ventricular dilatation.

(4) Grade IV: Accompanied by hemorrhagic infarction ventricular hemorrhage.

\subsubsection{Statistical Methods}

SPSS 19.0 statistical software for analysis, counting data with $t$ test.

\section{Results}

\subsection{General Information}

377 cases of intracranial hemorrhage of 1750 cases of children in this group, the prevalence rate of $21.5 \%$, Among which, 350 cases of intracranial hemorrhage occurred in 1315 premature infants, the prevalence rate of $26.6 \%(350 / 1315)$, 27 cases of intracranial hemorrhage in 435 term infants, the prevalence rate of $6.2 \%(27 / 435)$; The prevalence of preterm infants is significantly higher than that of term infants, the difference is statistically significant $(\mathrm{t}=3.27, \mathrm{P}<0.001)$.

\subsection{Bedside Brain Ultrasound Results}

Of 377 cases intracranial hemorrhage in children, the occurrence of PVH-IVH in 349 cases, 8 cases of subdural hemorrhage, subarachnoid hemorrhage in 15 cases, other parts of the bleeding in 5 cases. In 349 cases of PVH-IVH, grade I PVH-IVH had 232 cases $(66.5 \%)$. The sonogram was characterized by coronal incision in the precornu, and the vertical plane showed strong echo or slightly stronger echo at the caudal nucleus of the thalamus; there were 83 cases (23.8\%) of grade II PVH-IVH, and the ultra-sonographic features were the irregularities of the choroid plexus and the echo enhancement in the lateral ventricle triangle and posterior corners. 21 cases of grade III PVH-IVH, accounting for $6.0 \%$, ultrasound showed lateral orbital irregular or regular hematopoietic echo, with varying degrees of ventricular dilatation (Figure 1); Grade IV had 13 cases of PVH-IVH, accounting for $3.7 \%$. The ultra-sonographic features were ventricular dilatation with irregular strong echo, and spherical or fan-shaped strong echoes were seen above the lateral ventricle. The prevalence of high-risk newborns occur I level PVH-IVH was significantly higher than grade II $(\mathrm{t}=11.33)$, III level $(\mathrm{t}=16.61)$, IV stage $(\mathrm{t}=17.37)$, and there was significant difference between grade I and II, III and IV (P $<0.001)$

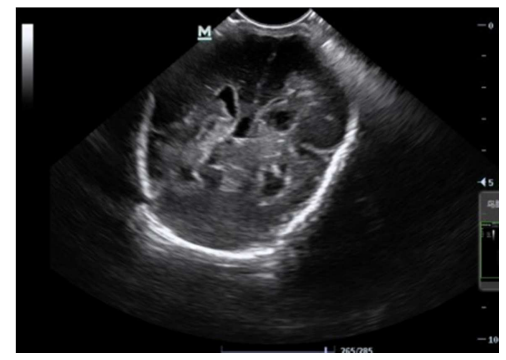

Figure 1. Ultrasound images of grade IV PVH-IVH.

\subsection{Comparison of the Coincidence Rate Between Bedside Brain Ultrasound and CT Diagnosis of Intracranial Hemorrhage}

All 349 cases of NICU diagnosed with PVH-IVH were examined by $\mathrm{CT}$ examination. And CT confirmed that there were 308 patients with PVH-IVH (Figure 2), 41 cases of bedside ultrasound diagnosis of ependymal hemorrhage (ie, grade I PVH-IVH) but they were not detected by CT, the detection rate was higher than $\mathrm{CT}$, bedside ultrasound and $\mathrm{CT}$ on intracranial hemorrhage detection rate was statistically significant $(\mathrm{T}=6.60, \mathrm{P}<0.001)$

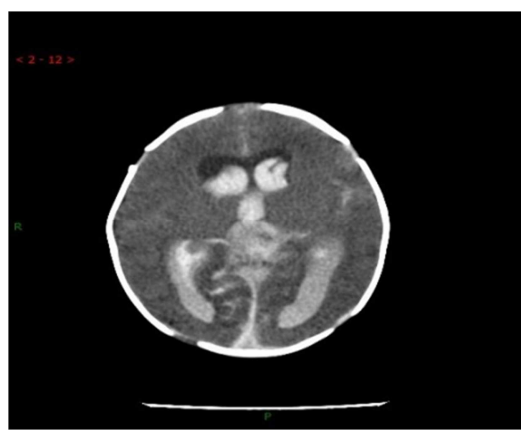

Figure 2. CT images of grade III PVH-IVH. 


\section{Discussion}

In the neonatal period, one of the most common intracranial lesions is intracranial hemorrhage, which is closely related to perinatal birth or asphyxia, leading to neonatal death or neurological developmental abnormalities during later period. Among intracranial hemorrhage, the most common is PVH-IVH. PVH-IVH can occur in the ependymal, intraventricular and periventricular [5], and preterm children are more likely to fall ill, thanks to premature infants with embryonic germinal maturation and internal and external factors in blood vessels. Fetuses in 24 to 32 weeks, the embryonic germinal layer of the matrix which originated in the caudate nucleus and thalamus junction, is composed of endothelial cells containing capillaries. Due to rich in mitochondria, metabolism are exuberant and they are in the active phase of the split, so they are vulnerable to hypoxia, acidosis and hypercapnia, resulting in necrosis, disintegration, bleeding. The larger the gestational age is, the less germination layer matrix is, and they will to disappear the full term [6]. Because of this feature, intracranial hemorrhage, especially the incidence rate of PVH-IVH is higher in premature children than full-term children.

In this group of patients, 41 cases of ultrasound showed subventricular hemorrhage (partial absorption was changed), but the CT test results were negative, showing that ultrasound detection of subventricular hemorrhage and a small amount of intraventricular hemorrhage has higher sensibility than CT [7]. Especially for I, II level PVH-IVH detection rate, ultrasound was significantly higher than the $\mathrm{CT}$ examination, which was conducive to follow-up intracranial hemorrhage prognosis. This group of results show that ultrasound diagnosis of PVH-IVH has a better sensitivity than CT, especially for the grade I PVH-IVH, the ultrasound diagnosis has the highest resolution, which is consistent with the literature reported [8].

Most of the intracranial hemorrhage confined to the ependymal membrane, that is, ependymal hemorrhage (grade I PVH-IVH), and ultrasound showed the sheet or group of echo-enhanced area can be seen in lateral anterior horn and the lower part of the body [9]. The border is still clear or less clear, and partially cystic degeneration; If the bleeding into the ventricle was II grade PVH-IVH, ultrasound can be found choroid plexus thickening, irregular, echo enhancement at this time, but there is no expansion of the ventricle. Children with I, II grade PVH-IVH has a state of consciousness which can be completely normal, and they are not accompanied by other symptoms or symptoms are extremely minor, so it is easy to overlook clinically, if not treated, it will bring serious sequelae to children with the course of progress. Therefore, early detection of neonatal intracranial hemorrhage is critical. Neonatal intracranial hemorrhage sequelae can be expressed as hydrocephalus, brain atrophy, and cystic changes and so on. Ultrasound has an extremely high sensibility for hydrocephalus, ependymal hemorrhage of the cyst, and the smallest cyst can be detected to about $2 \mathrm{~mm}$ diameter.

Ultrasound in the diagnosis of intracranial hemorrhage, there are still some limitations, this group of 16 cases diagnosed by CT but ultrasound missed, namely subdural hemorrhage in 5 cases, 4 cases of cerebellar hemorrhage, subarachnoid hemorrhage in 7 cases. Skull occlusion and acoustic window limitations affect the diagnosis of subdural hemorrhage [10]; posterior cranial fossa in the presence of echo reflection makes it very difficult to diagnose cerebellar hemorrhage with ultrasound; this group of ultrasound prompted 15 cases of subarachnoid hemorrhage, confirmed by $\mathrm{CT}$, but the other 7 cases missed, because the brain without echo is difficult to identify whether it is the blood or normal cerebral hemorrhage. Therefore, ultrasound diagnosis of subarachnoid hemorrhage is more difficult, such as the discovery of widening ditch or longitudinal pool and enhancing echo, we should be highly vigilant, and CT examination is recommended to avoid misdiagnosis. There are studies that CT is the of the best check means [2] to make diagnosis of subarachnoid hemorrhage. PVH-IVH occurs mostly in premature children, in the bedside brain ultrasound examination, we should pay attention to bilateral lateral ventricle anterior horn, white matter near the horn, lateral ventricle and dorsal white matter to observe whether there is obviously hyperechoic, hypoechoic and echoless, and whether dynamic review changes or not [7].

\section{Conclusion}

Due to CT examination's radiation, high inspection cost, use of sedation, and it can not be used bedside inspection. Besides, moving children will lead to greater fluctuations in blood pressure, which may cause changes in cerebral hemodynamics and is easy to induce PVH-IVH. Therefore, CT or MRI examinations are not advocated for premature children in the early postnatal period at home and abroad, what is promoted is bedside brain ultrasound. Bedside ultrasound with noninvasive, radioactive, economical, convenient and other advantages [11], it has a good application value in the newborn, so it should be used as a prenatal PVH-IVH routine screening items and dynamic monitoring methods [12], which provides a basis for clinical treatment. In addition, ultrasound can also be used to guide continuous lumbar puncture and efficacy evaluation of obstructive hydrocephalus after hemorrhage [13].

\section{References}

[1] Li Ling. High risk neonatal ultrasound diagnosis of traumatic brain injury and related factors [J]. Journal of Clinical Pediatrics, 2014, 32 (3): 295-296.

[2] Zhao Danxi, Zhao Yijiao, Guo Qinglu, et al. Clinical value of ultrasound and diagnosis of neonatal intracranial hemorrhage [J]. Chinese Maternal and Child Health Care, 2006, 21 (1): 58-60.

[3] Chinese Medical Association pediatric branch of neonatal school, Chinese Journal of Pediatrics editorial board. Preterm infants around the ventricle- intraventricular hemorrhage and diagnosis recommendations of softening periventricular white matter [J]. Chinese Journal of Pediatrics, 2007, 45 (1): 34-36. 
[4] Papile LA, Burstein $\mathrm{J}$, Burstein R, et al. Incidence and evolution of subependymal and intraventricular hemonhage: a study of infants with birth weights less than $1,500 \mathrm{gm}[\mathrm{J}]$. J Pediatr, 1978, 92 (4): 529-534.

[5] Liu Jing, Cao Haiying. Neonatal brain injury ultrasound diagnosis and clinical [J]. Beijing: China Medical Science and Technology Press, 2005: 86.

[6] Chen Wenmin, Ma Xiaoyan. Ultrasonographic diagnosis and analysis of intraventricular hemorrhage and intraventricular hemorrhage in high- risk neonates [J]. Journal of Medical Imaging Technology, 2011, 27 (8): 1551-1554.

[7] Li Zhian. Clinical ultrasound imaging [M]. Beijing: People's Health Publishing House, 2003: 1624.

[8] Li Xiao, Xian Jiang feng. Early diagnostic value of intraventricular hemorrhage and intraventricular hemorrhage in premature infants [J]. Journal of Medical Imaging, 2013, 21 (10): 749-751.
[9] Liu Jiaoran, Liu Fang, Peng Long, Zhang Hui, Cao Shikao, Zhang Chundong, Liu Hong, Du Zhifang. Clinical value of craniocerebral ultrasonography in the diagnosis of neonatal acquired brain injury [J]. Journal of Clinical Ultrasound in Medicine, 2016, (11): 770-772.

[10] He Weixuan, Pang Da. Clinical value of craniocerebral ultrasonography in the diagnosis of neonatal brain injury $[\mathrm{J}]$. Clinical Medical Engineering, 2014, (05): 553-554 +557.

[11] Maalouf EF, Duggan PJ, Counsell SJ, et al. Comparison of findings on cranial ultrasound and magnetic resonance imaging in preterm infants [J]. Pediatrics, 2001, 7:719-727.

[12] Wang Yongli. Ultrasound diagnostic value of neonatal ventricle-intraventricular hemorrhage $[\mathrm{J}]$. Chinese Journal of Practical Diagnosis and Therapy, 2011, 02: 175-176.

[13] Zhou Congle, Chen Huijin, Yu Renjie. Neonatal brain ultrasound diagnosis [M]. Beijing: Peking University Medical Press, 2007: 44. 